\title{
Reglamentación y uso de información privilegiada en el mercado norteamericano de valores ${ }^{1}$
}

\author{
MARIO LUIS PEROSSA², PABLO WALDMAN, DAMIÁN SERGIO DÍAZ UBERMAN ${ }^{4}$
}

\author{
UNIVERSIDAD DE BUENOS AIRES
}

\author{
Recibido, noviembre 04 de 2014 \\ Concepto evaluación, noviembre 24 de 2014 \\ Aceptado, abril 07 de 2015
}

Referencia: Perossa, M.L.; Waldman, P.; Díaz Ubermán, D.S. (2015). "Reglamentación y uso de información privilegiada en el mercado norteamericano de valores". Revista Academia y Virtualidad, 8, (1), 111-120

\section{Resumen}

El objetivo de este artículo de investigación se relaciona con el uso indebido de la información en los mercados de capitales, los cuales originan asimetría de la información y la selección adversa de aquellos que no tienen acceso o tienen acceso tardío a la información.

Aquí se presentan las regulaciones básicas del mercado norteamericano -la Securities Act de 1933 y la Securities Exchange Act de 1934 (Ley de Valores como comúnmente se la reconoce), la cual tiene dos objetivos primordiales: la creación de la SEC (Security and Exchange Commission), principal organismo responsable de la aplicación de la legislación de Estados Unidos en temas de valores federales, por un lado, y la regulación de los mercados secundarios, por el otro.

Concluye este cuerpo normativo la Investment Company Act de 1940 para la regulación de los mercados financieros de Estados Unidos. En este trabajo se desarrollan estos temas y se presentan algunos casos ocurridos que demuestran el efecto económico producido por las imperfecciones del mercado.

Palabras clave: asimetría de la información, información privilegiada, regulación del mercado, selección adversa.

1. Este artículo es un trabajo que forma parte de una serie de publicaciones relacionadas con un proyecto de investigación de la Universidad de Buenos Aires (UBA, PROINC, Expediente UBA 0024105/13, Resolución CD 3679/Investigación), tema: "Estrategias de inversión financiera en escenarios turbulentos". Director de la investigación: Mg. Mario L. Perossa; Investigadores: L. Q. Justo A. Mercado, L.E. Orlando D. Fortuna, L.E. Damián Díaz Uberman, L.A. Pablo Waldman y el alumno de la UBA-FCE estudiante de Actuario Martín Miliavsky

2. Magíster en Finanzas, Universidad Nacional de Rosario, Contador y Lic. en Administración, UBA.; profesor de grado y posgrado, UBA, UB y Universidad Maimónides. Contacto: perossa.mario@maimonides.edu

3. Licenciado en Administración, Universidad de Buenos Aires; docente UBA e Instituto Cámara Argentina de Comercio; participa en proyectos de investigación de la UBA. Contacto: pablo_waldman@hotmail.com

4. Licenciado en Economía, Universidad de Buenos Aires; docente, UBA; participa en proyectos de investigación de la UBA. Contacto: ddiazhospilab@ hotmail.com 
Reglamentación y uso de información privilegiada en el mercado norteamericano de valores

\section{Regulation and insider trading in the US stock market}

\section{Abstract}

The aim of this paper is exploring use of insider trading in the capital markets, producing information asymmetry and adverse selection for those who do not have access or otherwise delayed access to data. Here we can see the key regulations of the US market -Securities Act of 1933 and Securities Exchange Act of 1934, or Securities Act as it is commonly known, which has two main objectives: the foundation of SEC (Security and Exchange Commission), the main federal agency responsible enforcing the law of US financial markets and also the regulation of secondary markets. Finally, the Investment Company Act of 1940 to control US financial markets. These issues are addressed and other cases showing the economic impact by that market imperfection.

Keywords: asymmetrical information, insider trading, market regulation, adverse selection.

\section{Regulamentação e emprego de informação privilegiada no mercado norte-americano de valores}

\section{Resumo}

O escopo deste artigo de pesquisa relaciona-se com o emprego indevido da informação nos mercados de capitais, os quais originam assimetria da informação e seleção adversa daqueles que não têm aceso o aceso tardio à informação. Aqui se apresentam as regulações básicas do mercado norte-americano -a Securities Act de 1933 y la Securities Exchange Act de 1934 (Lei de Valores como comumente é conhecida), a qual tem dois alvos primordiais: a criação da SEC (Security and Exchange Commission), o principal organismo responsável da aplicação da legislação dos Estados Unidos no tema de valores federais, de um lado, e a regulação dos mercados secundários, do outro. Conclui este corpo normativo a Investment Company Act de 1940 para a regulação dos mercados financeiros de Estados Unidos. Neste trabalho desenvolvem-se esses temas e apresentam-se alguns casos que aconteceram e mostraram o efeito económico produzido pelas imperfeiçoes do mercado.

Palavras-chave: assimetria da informação, informação privilegiada, regulação do mercado, seleção adversa.

\section{Introducción}

Las leyes de valores federales de EEUU se instrumentan en una serie de estatutos, los cuales a su vez autorizan a una serie de regulaciones estatales promulgadas por la agencia gubernamental bajo la supervisión general -como el perro guardián federal de Wall Street- de la Securities and Exchange Commission. De hecho, existe una gran cantidad de reguladores y regulaciones que afectan a los emisores, los títulos y los brokers - de acuerdo con las características específicas de cada ente, el profesional que interviene en cada operación pueden estar obligado a cumplir a normas y reglamentos correspondientes a cada uno de los 55 organismos reguladores, incluyendo la Comisión de Valores existente en cada uno de los cincuenta estados más el Distrito de Columbia y Puerto Rico, además de la Securities and Exchange Commission, la National Association of Securities Dealers, Inc., y de las 
bolsas regionales en aquellos casos que corresponda.La Securities Act de 1933 es una de las principales leyes que brinda el marco jurídico necesario para el ordenamiento federal sobre emisiones de títulos, mientras que la Securities Exchange Act trata sobre la comercialización de títulos entre distintos inversores.

Cada una de las actas tiene una gran cantidad de normas y reglamentos de menores jerarquías promulgadas por las respectivas Comisiones de Bolsa y Valores. Uno de los objetivos centrales de las regulaciones de títulos y valores tiene como objetivo promover la divulgación material de la información requerida por mercados e inversores; esto significa incluir todos los aspectos de negociación del mercado y la financiación.

La apertura de la información permite a los inversores pero no al gobierno- realizar juicios sobre la viabilidad de los negocios de las distintas empresas y la posibilidad de comprar o no acciones, mientras que el gobierno federal - a través de la Securities Exchange Commission- requiere la exactitud -aunque no la garantiza- de la información proporcionada por la emisora.

\section{Marco teórico}

En opinión de Gibbins, M., Richardson, A., \& Waterhouse, J.,

[...] los incentivos económicos tanto para divulgar como para no divulgar información han sido identificados. Por un lado, los agentes quieren proteger la información de la que disponen para explotar sus potenciales ventajas económicas. Por el otro lado, los agentes podrían desear divulgar información financiera para aumentar el valor de la firma (1990).

La Securities Exchange Act de 1934, ley de valores o la ley del '34, como comúnmente se la reconoce, tiene dos objetivos primordiales: la creación de la SEC (Security and Exchange Commission -el principal organismo responsable de la aplicación de la legislación de los Estados Unidos en temas de valores federales- por un lado y la regulación de los mercados secundarios por otro. Fue "una de las primeras y, en opinión de muchos, una de las mejores leyes sancionadas bajo el marco del new deal" (Benston, G., 1973). Recordemos que

[...] La SEC realizó extensos estudios, incluyendo audiencias públicas, que revelaron una preocupación puntual, el desproporcionado efecto de la Securities Act de 1933 sobre los pequeños negocios que buscaban aumentar su capital mediante la venta de securities. Un estudio de la SEC confirmó la ampliamente aceptada visión de que, en base a sus activos, el costo de cumplir con los requisitos de registración del acta de 1933 eran sustancialmente mayores para los negocios pequeños que para los grandes. (Warren III, G, 1984).

La Investment Company Act de 1940 regula la organización de las empresas que se dedican principalmente en la inversión, la reinversión, y la negociación de valores, y cuyos valores propios son ofrecidos al público inversor.

Esta ley tiene como objetivo la revelación de la información material sobre el fondo de inversión y su perfil de inversión, sobre la forma de estructuración de la sociedad y las operaciones que el ente realiza.

\section{Metodología de la investigación}

La metodología empleada en el presente trabajo corresponde a una investigación teórica-descriptiva que combina casos prácticos reales tomados de la bibliografía legal que dieron las bases necesarias para luego dar lugar a las regulaciones legales correspondientes.

Es posible observar como en los mercados de capitales las regulaciones van detrás de los inversores, que ansiosos por ganancias extraordinarias hallan resquicios en la normativa para ejecutar estrategias que rozan lo ético y lo legal, operando en las zonas grises. No se hallaron papers que investiguen en forma integral los casos jurídicos con la emisión y regulación de leyes y normas. 
Reglamentación y uso de información privilegiada en el mercado norteamericano de valores

\section{Resultados y discusión}

Haciendo referencia al apetito por la generación de beneficios extraordinarios y la falta de regulación de anticipación, conviene rescatar el nacimiento de un mecanismo fraudulento de especulación que daría lugar a una terminología muy difundida en la jerga económica del siglo XX. La historia de Carlo Ponzi (Italia 1882, Brasil 1949), un inmigrante que fundó en 1919 una compañía que vendía cupones, prometiendo pagar beneficios del $50 \%$ a 45 días y del 100\% en 90 Días. Durante los primero meses el negocio funcionó de manera correcta atrayendo crecientes sumas de nuevos inversores, de tal forma que le permitía pagar las rentabilidades ofrecidas.

La situación dio cuenta de que Ponzi no invertía los fondos en la empresa, sino que pagaba las deudas emitiendo nuevas deudas. Conocida esta situación por los inversores, se desató una crisis de confianza que le impidió continuar con su "negocio" (Perossa, et al., 2012).

\section{Securities Act de 1933}

Mahoney, P. G. nos recuerda que

[...] La industria de los fondos de inversión enfrentaba obstáculos severos para influir en la arena legislativa en 1933. Su imagen pública se encontraba en su punto más bajo histórico. El nuevo presidente había usado exitosamente su discurso inaugural para asignarle públicamente a los financistas la culpa de la depresión y había hecho campaña prometiendo reducir su poder e influencia”. (2000).

Las consecuencias originadas de la crisis del '29, hicieron que los estados establezcan reguladores, que exijan y verifiquen el cumplimiento de mayores medidas de seguridad para el inversor, en especial el inversor no especializado, a través de mecanismos de información comparables. Esta situación es producto de los excesos del mercado. Si bien la intención primera es la protección del inversor común, tampoco quedan exentos los especialistas del mercado, por caso Irving Fisher afirmaba -antes de producirse el crash que "el país está marchando en una meseta alta de prosperidad..." para luego agregar "los precios de las acciones han alcanzado lo que parece ser una meseta alta permanente... Espero ver el mercado de valores mucho más alto que en la actualidad en unos pocos meses", Fisher fue un respetado académico, fundador y primer presidente de la Econometric Society que perdió gran parte de su prestigio y fortuna personal en el crash del 29'. Su infame frase citada marcó prácticamente el techo del mercado, que se derrumbó solo 3 días después. El gobierno de EEUU, al igual que los demás estados, siempre intentó restaurar la confianza de los inversores a través de mecanismos que significaran mayores obligaciones de información, de revelamiento (disclosure) de información obligatoria por parte de los emisores, como política esencial para reducir la asimetría de la información.

La Securities Act de 1933 está dirigida a los emisores de títulos valores, por lo tanto, su objeto de regulación está orientado al mercado primario de títulos, reconociendo la importancia de la divulgación oportuna en el valor de los títulos. Esta acta trata sobre los acuerdos de intercambio, los valores y las operaciones que se encuentran exentas, las prohibiciones relacionadas con el comercio de los títulos interestatales, la registración de esos títulos y la información requerida, incluido sus procedimientos y el detalle de la información que deberá ser revelada en el prospecto respectivo.

Da cuenta de la responsabilidad civil, de la prescripción de las acciones y las acciones en contrario; sobre la responsabilidad de los controladores y las acciones y el enjuiciamiento de los delitos, sobre la jurisdicción y las sanciones pertinentes. Concluye con la jurisdicción de otras agencias de gobierno relacionada con el control de los valores, los litigios sobre los valores privados y los conflictos de intereses relacionados con ciertas operaciones de securitización. Define al término security en general como cualquier instrumento generalmente llamado título o cualquier certificado de interés o participación -sea temporal o provisional- con derecho a suscripción o compra. En particular, security significa cualquier nota de tesorería, acciones, bonos, obligaciones o evidencias de deuda, participación en ganancias, contratos de inversión, 
opciones, participaciones transferibles, privilegio de ingresar en una bolsa de valores en relación con activos en moneda extranjera. Mientras que la ersona queda definida como cualquier persona física, corporación o asociación, un fideicomiso, una sociedad anónima $u$ organización política o una subdivisión de las mismas, así como también cualquier organización no constituida como sociedad.

Acerca de la información por revelar por parte del ente emisor, esta acta faculta a la comisión para que la misma adopte los reglamentos pertinentes, requiriendo que cada emisor de un activo debe revelar información por tramo o tipo de título y sobre los activos que respaldan la emisión, pues la Securities Act del '33 confía en la capacidad de los inversores para analizar la conveniencia y oportunidad de la decisión de inversión a realizar; por lo cual el objetivo que persigue esta regulación es el de brindar al inversor la información necesaria para que este pueda tomar la mejor opción.

La información debe ser adecuada respecto del emisor y puesta a disposición del público, indicando la naturaleza de los de los valores a registrar, revelando la estructura de capital del emisor, los derechos de los titulares a efectos del interés público y la protección de los inversores. Durante el proceso de registro, los emisores deben revelar información acerca de:

1. las propiedades de la empresa y los negocios;

2. las garantías que se ofrezcan para la venta;

3. información sobre la gestión de la empresa; y

4. estados financieros certificados por auditores independientes.

Si a juicio de la comisión encargada del registro, la misma considera que la información revelada por el emisor en la declaración de registro es falsa, o no menciona un hecho considerado material, o es engañosa, previa notificación al interesado y luego de oír su descargo, emitir una orden para detener o suspender la vigencia de la declaración de registro. Al momento de realizar la acción de detención de la declaración de registro, la comisión $-\mathrm{o}$ cualquier funcionario por ella designado- queda habilitada/o para exigir la presentación de todos los libros y documentos, pudiendo requerir la realización de un balance especial donde exhiba sus activos y sus pasivos o la declaración de rentas para ser certificados por un contador independiente o certificada y aprobada por la comisión. La falta de colaboración o la obstrucción por parte del emisor supone facultad para la emisión de una orden de detención de la declaración de registro.

Una definición importante llegado a este punto es el concepto de materialidad de la información, en la actualidad, las Normas Internacionales de Información Financiera (NIIF) definen el término material cuando las omisiones o afirmaciones erróneas, individuales o colectivas, pueden influenciar las decisiones que los usuarios hacen sobre la base de los estado financieros u otro tipo de información brindad por el ente emisor. La materialidad depende del tamaño y la naturaleza de la omisión o de la afirmación errónea valorada en las circunstancias correspondientes.

Los errores que no son considerados materiales deberían ser igualmente corregidos, ya que la suma de errores no materiales -por omisión o información equivocadapueden llegar a dar lugar a realizar afirmaciones erróneas materiales.

En función de proteger a los inversores del mercado de valores, la Securities Act contempla penalidades que van desde las multas hasta la prisión, de acuerdo con el carácter de la información brindada. Si la información expuesta en el informe de registro es materialmente falsa o contiene errores u omisiones materiales acerca del emisor, los valores o la transacción -o todos ellos-, entonces los inversores pueden iniciar demanda a los emisores y/o a los distribuidores de los títulos.

Resumiendo, la Securities Act de 1933 viene a dar marco a la revelación obligatoria de la información en forma oportuna de los emisores de títulos para los inversores y reguladores para las IPO (Initial Public Offerings), junto a la declaración de registro y las características del prospecto, con ejemplos que relacionan el concepto de 
Reglamentación y uso de información privilegiada en el mercado norteamericano de valores

materialidad; pero solo en el término de la emisión, o sea una sola vez, ya que está destinada al mercado primario (la emisión y colocación original de los títulos).

\section{Securities Exchange Act de 1934}

La ley de valores o la ley del '34, como comúnmente se la reconoce, tiene dos objetivos primordiales: la creación de la SEC (Security and Exchange Commission) -el principal organismo responsable de la aplicación de la legislación de los Estados Unidos en temas de valores federales- por un lado y la regulación de los mercados secundarios. El propósito de esta ley -a grandes rasgos- es proteger el comercio entre los distintos estados y el crédito a nivel nacional, como así también cumplir con el objetivo de asegurar el mantenimiento de la honestidad y justicia en los mercados de valores de EEUU.

La Comisión de Valores llevó a cabo importantes reformas al mandato de divulgación de la información en EE.UU. Para Beardsley, c. y O’Brien, j. (2004, p. 5) las regulaciones Norteamericanas de 1933/34 introdujeron un amplio criterio de reporte obligatorio.

Por ejemplo el enfoque a favor de los accionistas de la Securities Act de 1934 estableció un sistema de divulgación de información consistente en una combinación de reportes inmediatos, anuales y trimestrales"; el informe anual se realiza en el Formulario 10-K), los informes trimestrales en el Formulario 10-Q, y los informes actuales en el Formulario 8-K. Esta información hoy se encuentra en la base de datos EDGAR de la SEC

La sección 4 de la Securities Exchange Act de 1934 creó la Securities and Exchange Commission como una agencia del gobierno federal. Las responsabilidades inmanentes en materia judicial de la Comisión corresponden a jueces administrativos, mientras que los demás poderes administrativos pertenecen a sus cuatro divisiones y cinco oficinas regionales. Las divisiones de la Securities and Exchange Commission son:

1. División de Cumplimiento (Division of Enforcement), la cual se responsabiliza de investigar las violaciones a las leyes de valores. La SEC cuenta con acciones tanto de cumplimiento como administrativas, cuando existe una violación a las normas cometida por algún emisor, sus oficiales o empleados, broker-dealers.

2. División de Finanzas Corporativas (Division of Corporation Finance), la cual se encarga de revisar el cumplimiento de los documentos de registro con los requisitos de revelación de información especificados en la legislación de valores.

3. División de Regulación de Mercados (Division of Market Regulation), la cual expide las políticas que se vinculan con las bolsas de valores, los mercados OTC y los broker-dealers.

4. División de Administración de las Inversiones (Division of Investment Management), la cual se encarga de la regulación que corresponde al Investment Company Industry Act de 1940, el Investment Advisers Act de 1940, y el Public Utility Holding Company Act de 1935.

Al crear la Securities and Exchange Commission en 1934, el Congreso le otorgó a la comisión la responsabilidad en la administración de las leyes federales de valores; por lo tanto, el principal objetivo de la Securities and Exchange Commission corresponde a la ejecución y administración de dichas normas. La legislación federal de valores establece que la expedición de reglas por parte de la SEC deberá estar orientada a la protección a los inversionistas y la promoción de la eficiencia, competencia y formación del capital.

\section{La aplicación de las leyes federales. EI caso USA vs O'Hagan (1997)}

Una de las reglas más relevantes establecida por dicha ley se trata de la sección 10, regla 10b-5, formalmente conocida como Empleo de prácticas manipulativas y/o engañosas, cuyo campo de acción de la norma incluye a cualquier persona que directa o indirectamente defraude, realice declaraciones falsas, omita información relevante o conduzca cualquier tipo de operación comercial que 
engañe a otra persona ya sea directa o indirectamente; en relación a transacciones que involucren acciones o instrumentos financieros.

Es una sección de la ley que fue redactada en términos muy amplios y ha sido aplicada incluso en casos de fraude internacional. Aunque estos casos generan dudas sobre el alcance jurisdiccional de la ley norteamericana y presentan incluso problemas delicados a nivel diplomático, la SEC no ha vacilado en actuar fuera de sus fronteras cuando lo cree necesario para la protección del público inversor (Loomis, 1978)

Un caso paradigmático que amplió los límites de aplicación de la ley en lo relativo al uso de información privilegiada se dio en el caso USA vs O’Hagan (1997) ya que el acusado no estaba directamente involucrado en ninguna de las compañías que realizaron la transacción por la que se obtuvieron los beneficios. James O'Hagan se desempeñaba como abogado en el prestigioso bufete Dorsey \& Whitney cuando este mismo fue contratado por la empresa Grand Metropolitan PLA, quien estaba estudiando la posibilidad de realizar una oferta pública de adquisición por Pillsbury Company. O’Hagan aprovechó su conocimiento privilegiado para comprar una gran cantidad de opciones sobre los valores de Pillsbury. Una vez que la adquisición fue anunciada públicamente, O'Hagan se desprendió de las mismas obteniendo una ganancia de U\$S 4.3 millones.

La Corte suprema condenó a O'Hagan de 57 cargos de fraude porconsiderarque tenía un deberdeconfidencialidad con quienes le suministraron la información, ya que la misma le fue otorgada con cierto fin preciso y fue utilizada para provecho propio. La Corte Suprema denominó a esta ruptura del acuerdo de confidencialidad como "fraude sobre la fuente de información" aplicando la tesis de la apropiación inadecuada (Misappopiation Theory).

Según esta teoría, una persona comete fraude en conexión con una transacción de valores cuando utiliza información confidencial quebrantando el compromiso debido a su fuente de información. Esta interpretación permite condenar a personas externas a las compañías (outsiders) tales como el asesor O'Hagan y se complementa con la teoría denominada clásica que se centra en quienes están involucrados activamente en la empresa (insider) y por lo tanto violentan la confianza que los accionistas depositan en ellos.

\section{Ley de Sociedades de Inversión de 1940 (Investment Company Act de 1940)}

La Investment Company Act de 1940 regula la organización de las empresas que se dedican principalmente en la inversión, la reinversión, y la negociación de valores, y cuyos valores propios son ofrecidos al público inversor. Esta ley tiene como objetivo la revelación de la información material sobre el fondo de inversión y su perfil de inversión, sobre la forma de estructuración de la sociedad y las operaciones que el ente realiza. La ley define como "Sociedad de inversión" a cualquier entidad cuya actividad principal esté relacionada con el negocio de la inversión, la reinversión, o la negociación de valores y pueda emitir certificados para participar al público inversor en el negocio en forma de cuota, o -como último caso- se dedica a la inversión, la reinversión, explotación, o negociación de valores, y es propietaria o tiene intención de poseer títulos de inversión.

El reglamento está diseñado para minimizar los conflictos de intereses que surjan en estas operaciones complejas, por lo tanto la Ley exige a las empresas a revelar su situación financiera y las políticas de inversión a los inversores e interesados. Es importante recordar que la Ley no permite a la SEC supervisar directamente las decisiones de inversión o actividades de estas empresas o juzgar los méritos de sus inversiones. La Investment Company Act de 1940 (la "Ley de Sociedades de Inversión") es la ley que ofrece el marco regulatorio a la industria de los hedge funds.

\section{Investment Advisers Act de 1940}

La Investment Advisers Act de 1940 persigue el fin de controlar a aquellos que, por el pago de una cuota, asesoran a personas, fondos de pensiones, y las demás organizaciones en temas relacionados con inversiones 
Reglamentación y uso de información privilegiada en el mercado norteamericano de valores

financieras. Se entiende a un asesor de inversiones a una persona física o ideal que presta asesoramiento de inversión a cambio del pago de una cuota. Se consideran a los asesores de inversión como de interés nacional, por lo cual se plantea que:

1. Sus consejos, asesoramiento, publicaciones, escritos, análisis, informes son distribuidos, y sus contratos, acuerdos de suscripción, y otros arreglos con los clientes se negocian y se realizan utilizando el correo y los medios e instrumentos del comercio interestatal.

2. Sus consejos, asesoramiento, publicaciones, escritos, análisis e informes habitualmente se relacionan con la compra y venta de valores negociados en las bolsas de valores nacionales y en mercados interestatales Over the counter, los valores emitidos por empresas dedicadas al negocio en el comercio interestatal, y valores emitidos por bancos nacionales y los bancos miembros del Sistema de Reserva Federal, y

3. Las operaciones anteriores se producen en el volumen suficiente como para afectar sustancialmente el comercio interestatal, bolsas de valores nacionales, $\mathrm{y}$ otros mercados de valores, el sistema bancario y la economía nacional.

Al margen de algunas pocas excepciones, la Investment Advisers Act de 1940 requiere que las empresas o profesionales independientes que cobran por sus servicios de asesoramiento a otros sobre las inversiones en valores deben registrarse ante la SEC y se ajustan a las regulaciones diseñadas para proteger a los inversores.

\section{La Securities and Exchange Commission, y} establece normas para la publicidad, divulgación, derechos, responsabilidad, y el mantenimiento de registros. La Investment Advisers Act de 1940 tiene por objeto la protección del público inversor.

Es un delito para los asesores de inversión proporcionar información falsa o engañosa a sus clientes. Mathews (1971) opina que la ley se diseñó en parte para establecer amplios estándares que le permitan al gobierno condenar por operaciones en beneficio propio a personas afiliadas a compañías de inversión, una industria que por su propia naturaleza dificulta particularmente obtener pruebas concretas

Quedan excluidos de la ley aquellos profesionales que ofrecen asesoría de inversión a sus clientes de manera incidental. En el Investment Advisers Act de 1940 se excluye "a cualquier abogado, contador, ingeniero o maestro cuya prestación de servicios se realice exclusivamente relacionados con el ejercicio de su profesión".

\section{Conclusiones}

Las bolsas de comercio y sus mercados de valores asociados tienen como principal objetivo permitir la canalización y el direccionamiento eficiente de flujos de fondos de inversión desde los inversionistas institucionales y particulares hacia las empresas que buscan fondearse a bajas tasas.

A pesar de trabajar en mercados organizados, con contraparte central y las regulaciones sobre las revelaciones a que están obligadas las empresas; las relaciones que se crean entre los inversionistas, los intermediarios -como lo agentes de bolsa- y los emisores de títulos valores se ven afectadas por asimetrías de información que encarecen la tasa de interés encareciendo los costos de financiamiento. Si bien el problema de la información asimétrica se reduce mediante la regulación que se proponen los estados y entes reguladores desde las normas y leyes que se establecen y supervisan tanto por las agencias gubernamentales como por las propias bolsas de valores.

Son muchos los factores que se deben considerar a la hora de diseñar y evaluar un adecuado marco regulatorio para la efectiva operación de un mercado de valores. Preservar la competencia y proteger a los inversionistas son, probablemente, los principales objetivos de un adecuado marco regulador. Además, la legislación y regulación debe promover el desarrollo del mercado y no dictar la forma en la que éste debe desarrollarse. Este problema de información asimétrica se atenúa mediante la interacción de diversas instituciones y leyes que dan a 
los inversionistas información relativamente confiable de la calidad de los diferentes emisores y sus intermediarios. Las normas sobre regulación de la actividad financiera en los mercados de valores de EEUU se nutren de un sinnúmero de normas menores controladas por una enorme cantidad de agencias estatales, siendo el principal cuerpo normativo rector de esta actividad las que se mencionaron en el trabajo. Ellas le proveen -a la SEC en especial-de las herramientas necesarias para controlar los movimientos de los flujos de inversiones y ofrecer ciertos estándares de calidad y seguridad en la información ofrecida al mercado, buscando reducir los niveles de la asimetría propia de los mercados, castigando el uso indebido y facilitando la toma de decisiones racionales reduciendo los niveles de selección adversa de los inversores no institucionales.

También resulta observable la capacidad y voracidad de los distintos actores del mercado por hacerse de beneficios derivados del uso de información privilegiada, dando lugar a los reguladores del mercado para administrar justicia llevando a juicio a aquellos que hacen abuso de su situación de privilegio. Resulta importante la adecuación constante y permanente de las leyes para lograr un conjunto de normas adecuadas al efecto y actualizadas para prevenir el fraude asociado a las nuevas formas de utilización de información privada no divulgada. Es conocida la experiencia que en ausencia de una apropiada política de regulación y supervisión de los participantes de los mercados de valores no es posible promover una clara competencia ni tampoco garantizar una adecuada protección a los inversionistas. El análisis de la regulación en los países centrales indica que la tarea es muy laboriosa e intrincada, pero remarca la necesidad que su regulación y supervisión deba realizarse coordinadamente entre las agencias gubernamentales pertinentes y las propias bolsas de comercio y mercados de valores.

El desarrollo del mercado de valores es uno de los principales impulsores del crecimiento económico de un país, permitiendo la financiación de proyectos de inversión de las compañías por una parte y ofreciendo alternativas de inversión para distintos niveles de apetito de riesgo de los inversores. El desarrollo de un mercado de capitales incipiente se hace importante cuando se les garantiza a los inversores una adecuada protección -por ejemplo a los accionistas minoritarios- y se definen políticas claras de competencia de los reguladores para habilitar las condiciones para una competencia transparente entre los participantes del mercado.

La línea investigativa del presente trabajo indaga sobre el comportamiento de los inversores y la respuesta tardía-de los reguladores del mercado para evitar nuevas estrategias y argucias en zonas débilmente reguladas para sacar provecho en su propio beneficio. Algo llamativo durante la investigación es el hecho de que los reguladores provienen en su mayoría del mercado, y luego en algún momento vuelven al mercado nuevamente como inversores institucionales o en cargos importantes en compañías de inversión. Una línea de investigación importante que queda plasmada responde a los siguientes interrogantes: los ejecutivos que pasan a ser reguladores: ¿Se desentienden plenamente de sus relaciones con los clientes? ¿Son tan perspicaces como en el campo privado? ¿Qué los motiva a pasar al terreno público?

\section{Referencias}

Beardsley, C. y O'Brien, J. (2004) "Measuring the impact of regulation on Market Stability: Evidence from the USA markets" ISMA center discussion papers in finance.

Benston, G. J. (1973). Required disclosure and the stock market: An evaluation of the Securities Exchange Act of 1934. The American Economic Review, 132-155.

Cachanosky, J. C. "La crisis del treinta", Revista Libertas VI: 10, Instituto Universitario ESEADE, Buenos Aires, mayo de 1989.

Day, R. (2000). "UK Accounting regulation, a historical perspective" Bournemouth University.

Dewing, I.P. and Russell, P.O. (2004), Regulation of UK Corporate Governance: lessons from accounting, audit and financial services. Corporate Governance: An International Review, 12 
Reglamentación y uso de información privilegiada en el mercado norteamericano de valores

Gibbins, M., Richardson, A. \& Waterhouse, J. (1990). The management of corporate financial disclosure: opportunism, ritualism, policies, and processes. En: Journal of accounting research, 121-143.

Higgs, R. (1997). "Regime Uncertainty. Why the Great Depression Lasted so Long and Why Prosperity Resumed after the War" The Independent Review, Vol. I, No 4,

Loomis Jr., P.A., \& Grant, R. W. (1978). US Securities and Exchange Commission, Financial Institutions Outside the US and Extraterritorial Application of the US Securities Laws, The. J. Comp. Corp. L. \& Sec. Reg., 1, 3.

Mahoney, P.G. (2000). The political economy of the Securities Act of 1933.UVA Law School, Legal Studies Working Paper No. 00-11.

Mathews, A.F. (1971). Criminal Prosecutions Under the Investment Company Act of 1940 and the Investment Advisers Act. BC Indus. \& Com. L. Rev., 13, 1257.

Obstfeld M. and Taylor A. (1997). "The great depression as a watershed: International Capital Mobility over the long run" Working paper 5960. National Bureau of Economic Research.

Painter, R.W., Krawiec, K.D., \& Williams, C.A. (1998). Don’t Ask, Just Tell: Insider Trading After United States v. O’Hagan. Virginia Law Review, 153-228.

Perossa, M.; Díaz Uberman, D. \& Waldman, P. (2012) Crisi y Regulación en los Mercados Financieros. En los países centrales, desde 1030 a nuestros días. AV Akademikerverlag GmbH \& Co. KG. Saarbrücken, Deuschland, 33-34.

Pritchard, A.C. (1998). United States v. O'Hagan: Agency Law and Justice Powell's Legacy for the Law of Insider Trading. BUL Rev., 78, 13.
Rauchway, E. (2008). "The Great Depression \& the New Deal, A Very Short Introduction" Oxford University Press

Robb Victoria (1997), "The Genesis of Regulation". Financial Stability Review. Autumn 97’.

Romer, C (1988). "The great crash and the onset of the great depression”. Working paper 2639. National Bureau of Economic Research.

Rose, N.; Joskow, P. (1989) “The Effects Economic regulation”, handbook of Industrial Organizations, Volume II, editorial: R.D. Willig@Elsevier Science Publishers B. V.

Scott, K.E. (1980). Insider trading: rule 10b-5, disclosure and corporate privacy. J. Legal Stud., 9, 801.

Ulph Janet, "Commercial Fraud. Civil liability, human rights and money laundering” Oxford University Press, 2006.

Vitols, Sigurt, "From Banks to Markets: The Political Economy of Liberalization of the German and Japanese Financial Systems", Wissenschaftszentrum Berlin für Sozialforschun, 2001.

. "The Origins of Bank-Based and Market-Based Financial Systems: Germany", Japan and the United States, in The origins of nonliberal Capitalism: Germany and Japan, edited by W. Streeck and K. Yamamura. U. of Cornell Press.

Wang, W.K. (1980). Trading on Material Nonpublic Information on Impersonal Stock Markets: Who is Harmed, and Who Can Sue Whom Under SEC Rule 10b-5. S. Cal. L. Rev., 54, 1217.

Warren III, G. (1984). “A Review of Regulation D: The present exemption regimen for limited offerings the Securities Act of 1933". En: The American University Law Review, Volume 33. 